\title{
Crystalline beams in dispersion-free storage rings
}

\author{
Masahiro Ikegami \\ Institute for Chemical Research, Kyoto University, Gokasho, Uji, Kyoto 611-0011, Japan \\ Hiromi Okamoto \\ Graduate School of Advanced Sciences of Matter, Hiroshima University, 1-3-1 Kagamiyama, Higashi-Hiroshima, \\ Hiroshima 739-8530, Japan \\ Yosuke Yuri \\ Takasaki Advanced Radiation Research Institute, Japan Atomic Energy Agency, 1233 Watanuki-machi, Takasaki, \\ Gunma 370-1292, Japan
}

(Received 9 August 2006; published 7 December 2006)

\begin{abstract}
Generating a multidimensional crystalline beam in a storage ring has been known to be difficult without a special cooling force, i.e., tapered cooling, because of the momentum dispersion induced by bending magnets. It is, however, possible to eliminate the dispersion all around the ring by adding an electric dipole field in each magnetic bending region. A storage ring with such unique deflectors should enable us to reach multidimensional crystalline states with an ordinary untapered cooling force. In order to verify this expectation, molecular dynamics simulations are performed to study beam crystallization in several dispersion-free storage rings including the S-LSR at Kyoto University. The present results show that various crystalline states can be established without relying on the tapered force.
\end{abstract}

DOI: 10.1103/PhysRevSTAB.9.124201

PACS numbers: 29.20.Dh, 41.75.-i, 52.59.Sa

\section{INTRODUCTION}

In the ultimate low temperature state of an ion beam, the perfect balance is achieved between the external focusing force and internal Coulomb repulsive force. The ions form an ordered structure where their relative motions are nearly frozen out in the beam rest frame [1-5]. Such a new state of matter is called a crystalline beam whose transverse emittances are ideally equal to zero except for quantum noise. In an actual accelerator, the beam receives discrete focusing forces from quadrupole magnets and deflective forces from dipole magnets. This realistic situation leads to two stringent criteria for attaining a crystalline beam. According to Refs. [6,7], we need a strong-focusing machine that operates below the transition energy. It has also been pointed out that the phase advance of the singleparticle betatron oscillation must be below 127 degrees per lattice period [8-10]. In practice, these two requirements can be met by designing a proper storage ring. Besides them, at least two additional conditions are known which should be satisfied by the cooling force. First, the cooling rate must be high enough to overcome heating from intrabeam scattering. Although the laser cooling force, which is currently the only means toward beam crystallization, is one-dimensional (1D), we can readily make it work in all three directions by applying the resonant coupling method [11,12]. Second, the cooling force must have such nature as to drive the beam into a stationary state where all particles have the same angular velocity in the ring. Once a beam is crystallized, the path length of a particle traveling along a radially outer orbit becomes longer than those of inner particles; in other words, the outer particle has to run slightly faster than the inner particles, so the revolution frequencies are identical. Regular cooling forces are not suitable for this specific character of a crystalline state because they simply equalize the linear particle velocity at the cooling section. Multidimensional crystals are then exposed in bending regions to shear that seriously affects the beam stability. $\mathrm{We}$, therefore, need the so-called tapered force to establish stable crystalline states in a storage ring $[9,13]$. The ideal tapered dissipation can be formulated as [14]

$$
\Delta p_{z}=-f_{z}\left(p_{z}-C_{x s} x / \rho\right)
$$

where $p_{z}$ is the scaled longitudinal momentum of a particle in the beam rest frame, $\rho$ is the radius of curvature in the bending regions, the left-hand side represents the change in $p_{z}$ at the cooling section, $f_{z}$ is the strength of the cooling force, and $C_{x s}$ is the tapering factor dependent on the lattice structure of the storage ring. The optimum value of the tapering factor can be determined by solving coupled differential equations similar to the well-known beam envelope equations [15]. It is evident from Eq. (1) that the longitudinal average velocity of each particle is linearly proportional to the horizontal coordinate $x$ in the final equilibrium state reached by the tapered force. The tapered cooling can thus realize all kinds of crystalline configurations if the ring lattice satisfies the conditions mentioned above [16]. The ideal tapering factor is, however, not constant but varies along the beam orbit. Since the cooling section extends over some distance, the perfect matching of the tapering factor is probably impossible in practice 
whereas it is very important to adjust its magnitude for the stabilization of a large crystalline beam [16].

An alternative idea to equalize the angular velocities of stored particles was first proposed by Pollock [17]. His idea is to use a horizontal electric field perpendicular to a magnetic dipole field in each bending region. Since the scalar potential of the electrostatic field accelerates or decelerates particles at the edges of the deflection element, the particles can have the same angular velocity in the bending section and accordingly receive no shear force. This device has been known as a dispersion-free deflector for mass spectrometry $[18,19]$. The beam motion in a storage ring with the dispersion suppressor is governed by a Hamiltonian almost equivalent to that for a linear Paul trap where Coulomb crystallization can be realized easily [20].

In this paper, we numerically explore the beam behavior in dispersion-free rings, applying the molecular dynamics (MD) technique. In Sec. II, we show the basic equations of motion in the beam rest frame for MD simulations. The lattice structures assumed in the present simulations are outlined in Sec. III. The MD code CRYSTAL [21] is employed in Sec. IV to show that multidimensional crystalline beams can be generated in dispersion-free rings without tapering the cooling force. The structural transition of coasting and bunched crystalline beams is studied in Sec. V. Section VI is devoted to practical considerations of cooling experiments at S-LSR where the dispersion suppressor has been actually implemented. Finally, the present results are summarized in Sec. VII.

\section{EQUATIONS OF MOTION IN THE BEAM REST FRAME}

In order to apply the MD algorithm, it is convenient to observe the dynamic motions of particles in the beam rest frame whose origin circulates around the storage ring. The effect of radiation at bends can be ignored because we only consider low-energy heavy ion beams. The three axes are taken such that they are always oriented to the radial, vertical, and tangential directions. In this system, the particle motion becomes nonrelativistic, which considerably simplifies the numerical computation. This effort was first made by Wei, Li, and Sessler [6,7]. We here somewhat generalize their approach, introducing the effect of electrostatic deflectors. Provided the gravitational force is negligible, the equations of motion can be written in a general system of coordinates as

$$
\frac{D P_{i}}{d \tau}=F_{i}, \quad F_{i} U^{i} \equiv 0,
$$

where we have adopted the notations of Møller [22]. In the present case, the nongravitational four force $\left\{F_{i}\right\}$ originates from the electromagnetic fields and satisfies the relation $F_{i}=(e / c) F_{i k} U^{k}$, where $e$ is the electric charge of the particle, and $c$ is the speed of light. Suppose that, in the laboratory frame, the radius of curvature in a dispersionfree bend is $\rho$ and the dipole magnet has a uniform field of flux density $B_{0}$. The scalar potential within the bend is then given by

$$
\phi_{D}(X, Y)=-V_{0}\left[\frac{X}{\rho}-\frac{n}{2}\left(\frac{X}{\rho}\right)^{2}-\frac{(1-n)}{2}\left(\frac{Y}{\rho}\right)^{2}+\cdots\right]
$$

where $V_{0} / \rho$ corresponds to the strength of the dipole electric field, and $(X, Y)$ are the transverse coordinates in the Frenet-Serret system set along the design beam orbit. The field index $n$ has been defined by $n \equiv-\rho / E_{X}$. $\partial E_{X} /\left.\partial X\right|_{X, Y=0}$, where $E_{X}$ is the horizontal electric field given by $E_{X}=-\partial \phi_{D} / \partial X$. We now introduce the rectangular rotating coordinate system $(x, y, z, c \tau)$ whose origin is fixed at the position of the reference particle. $\tau$ is the proper time in the beam rest frame. The $z$-axis points toward the direction tangent to the design orbit while $x$ and $y$ denote the radial and vertical coordinates. Moving to this system, we obtain the following linearized electromagnetic fields after considerable algebra [23]:

$$
\begin{gathered}
E_{x}^{\prime} \approx \gamma V_{0}\left(\frac{1}{\rho}-\frac{n x}{\rho^{2}}\right)-\beta \gamma B_{0}\left(1+\frac{x}{\rho}\right), \quad E_{y}^{\prime} \approx-\frac{\gamma V_{0}(1-n)}{\rho^{2}} y, \quad E_{z}^{\prime} \approx \frac{\gamma^{3}}{\rho}\left(\frac{V_{0}}{\rho}-\beta B_{0}\right) z, \\
B_{x}^{\prime} \approx \frac{\beta \gamma V_{0}(1-n)}{\rho^{2}} y, \quad B_{y}^{\prime} \approx \gamma B_{0}-\beta \gamma V_{0}\left(\frac{1}{\rho}-\frac{n x}{\rho^{2}}\right), \quad B_{z}^{\prime}=0,
\end{gathered}
$$

where $\beta$ and $\gamma$ are the Lorentz factors of the reference particle, and space charge fields have been dropped for brevity. The metric tensor of the beam rest frame can be written as

$$
g_{i j}^{\prime}=\left(\begin{array}{cccc}
1 & 0 & 0 & -\beta \gamma^{2} z / \rho \\
0 & 1 & 0 & 0 \\
0 & 0 & 1 & \beta \gamma^{2} x / \rho \\
-\beta \gamma^{2} z / \rho & 0 & \beta \gamma^{2} x / \rho & \gamma^{2}\left[\beta^{2}(1+x / \rho)^{2}+(\beta \gamma z / \rho)^{2}-1\right]
\end{array}\right) .
$$

Making use of the field components (4) and metric tensor (5) in Eq. (2) and taking the Coulomb interactions into account, we finally reach the equations of motion, 


$$
\left\{\begin{array}{l}
\ddot{x}=-\left[(1+n) \frac{e V_{0}}{\beta^{2} E_{0}}-\gamma^{2}+1\right] \frac{x}{\rho^{2}}+\left(1-\frac{e V_{0}}{\beta^{2} \gamma^{2} E_{0}}\right) \frac{\gamma \dot{z}}{\rho}-\frac{r_{p}}{\beta^{2} \gamma^{2}} \frac{\partial \phi_{c}}{\partial x}, \\
\ddot{y}=-(1-n) \frac{e V_{0}}{\beta^{2} E_{0}} \frac{y}{\rho^{2}}-\frac{r_{p}}{\beta^{2} \gamma^{2}} \frac{\partial \phi_{c}}{\partial y}, \\
\ddot{z}=-\left(1-\frac{e V_{0}}{\beta^{2} \gamma^{2} E_{0}}\right) \frac{\gamma \dot{x}}{\rho}-\frac{r_{p}}{\beta^{2} \gamma^{2}} \frac{\partial \phi_{c}}{\partial z}
\end{array}\right.
$$

where $r_{p}$ is the classical particle radius defined by $r_{p} \equiv$ $e^{2} / m_{0} c^{2}$ with $m_{0}$ being the rest mass, $E_{0} \equiv m_{0} \gamma c^{2}$ is the energy of the reference particle in the laboratory frame, the dot stands for differentiation with respect to the path length $s=\beta \gamma c \cdot \tau$ along the design orbit, and $\phi_{c}$ is the scaled Coulomb potential

$$
\phi_{c}=\sum_{j} \frac{1}{\sqrt{\left(x_{j}-x\right)^{2}+\left(y_{j}-y\right)^{2}+\left(z_{j}-z\right)^{2}}} .
$$

Here, the summation is performed over all the other particles $j$ and their image charges. These equations can be derived from the Hamiltonian

$$
\begin{aligned}
H= & \frac{p_{x}^{2}+p_{y}^{2}+p_{z}^{2}}{2}-\frac{\gamma}{\rho}\left(1-\frac{e V_{0}}{\beta^{2} \gamma^{2} E_{0}}\right) x p_{z} \\
& +\frac{1}{2}\left[1-(1-n) \frac{e V_{0}}{\beta^{2} E_{0}}+\frac{1}{\gamma^{2}}\left(\frac{e V_{0}}{\beta^{2} E_{0}}\right)^{2}\right] \frac{x^{2}}{\rho^{2}} \\
& +\frac{1}{2}(1-n) \frac{e V_{0}}{\beta^{2} E_{0}} \frac{y^{2}}{\rho^{2}}+\frac{r_{p}}{\beta^{2} \gamma^{2}} \phi_{c},
\end{aligned}
$$

where $p_{x}, p_{y}$, and $p_{z}$ are the scaled momenta conjugate to the coordinates $(x, y, z)$. It is worthy to recognize that Eq. (8) is identical to the Hamiltonian obtained from the standard beam-dynamics formalism [20]. The cross term $x p_{z}$ in Eq. (8), the most essential difference between a storage ring and an ion trap, is responsible for the shear effect that may destroy ordered structures at ultralow temperature. However, we now have an additional electric cross term due to the introduction of the deflector. By properly choosing the strength of the deflective electric field, it is possible to achieve $e V_{0} / \beta^{2} \gamma^{2} E_{0}=1$, which totally eliminates the cross term. The situation then becomes physically equivalent to that in an ion trap where various Coulomb crystals can be formed with the conventional laser cooling technique.

\section{SIMULATION PARAMETERS}

A storage-ring lattice appropriate for beam crystallization must fulfill the two conditions [6,9]; namely, for crystal formation,

$$
\gamma<\gamma_{t}
$$

and for crystal maintenance [24],

$$
N_{\text {sp }}>2 \sqrt{\nu_{x}^{2}+\nu_{y}^{2}}
$$

where $\gamma_{t}$ is the transition energy, $N_{\mathrm{sp}}$ is the lattice superperiodicity of the ring, and $\left(\nu_{x}, \nu_{y}\right)$ are the betatron tunes in the absence of space charge effects. When $\nu_{x} \approx \nu_{y}$, the latter condition becomes $\nu_{x(y)}<N_{\mathrm{sp}} / 2 \sqrt{2}$ that agrees with the original expression given in Refs. [8,9]. Note that the maintenance condition in Eq. (10) is closely related to the low-order resonant instability that depends on beam temperature. We can actually prove that this condition is relevant only in the crystalline regime. In order to prevent the occurrence of dangerous linear resonance in the whole temperature region throughout the cooling process, we demand

$$
\nu_{x(y)}<\frac{N_{\mathrm{sp}}}{4}
$$

instead of $\nu_{x(y)}<N_{\mathrm{sp}} / 2 \sqrt{2}$ [25].

For a systematic study of beam crystallization, lattice structures employed for simulations should meet the two requirements Eqs. (9) and (10) [preferably Eq. (11)]. We here consider three model rings whose bending regions contain the dispersion-suppressing electrodes. The lattice functions across a single focusing period are shown in Fig. 1. While the betatron tunes in the regular operating mode and in the dispersion-free mode have been set equal, the corresponding lattice functions are rather different. Other main parameters are summarized in Table I indicating that the necessary conditions have been satisfied in all three cases. The condition of crystal formation, i.e., Eq. (9), is automatically fulfilled in the dispersion-free operation [20]. The betatron tunes of Test Ring I and Test Ring II, both of which are designed solely for this study, have been set below $N_{\text {sp }} / 4(=2.5)$. S-LSR is the cooler storage ring constructed at Kyoto University [26]. This ring is being equipped with a laser cooling system and actually has the dispersion suppressor. The tunes can be adjusted below $N_{\mathrm{sp}} / 2 \sqrt{2}(=2.12)$ but, unlike the other two rings, the condition (11) cannot be met in the dispersionfree operation because the electrode potential enhances transverse focusing. Although it is preferable to have both tunes below $N_{\text {sp }} / 4$ rather than $N_{\text {sp }} / 2 \sqrt{2}$, we can establish stable crystalline states at S-LSR with the current parameters, provided that the line density of the beam is low and sufficiently strong cooling forces are available in all three directions. In what follows, we assume the "untapered" linear friction $\Delta p_{z}=-f_{z} p_{z}$ unless explicitly noted. Transverse linear cooling forces $\Delta p_{x(y)}=$ $-f_{x(y)} p_{x(y)}$ are also employed to reach perfect crystalline states. These dissipative forces are simultaneously applied to ${ }^{24} \mathrm{Mg}^{+}$ions circulating at the total kinetic energy of $35 \mathrm{keV}$. 
(a) Test Ring I
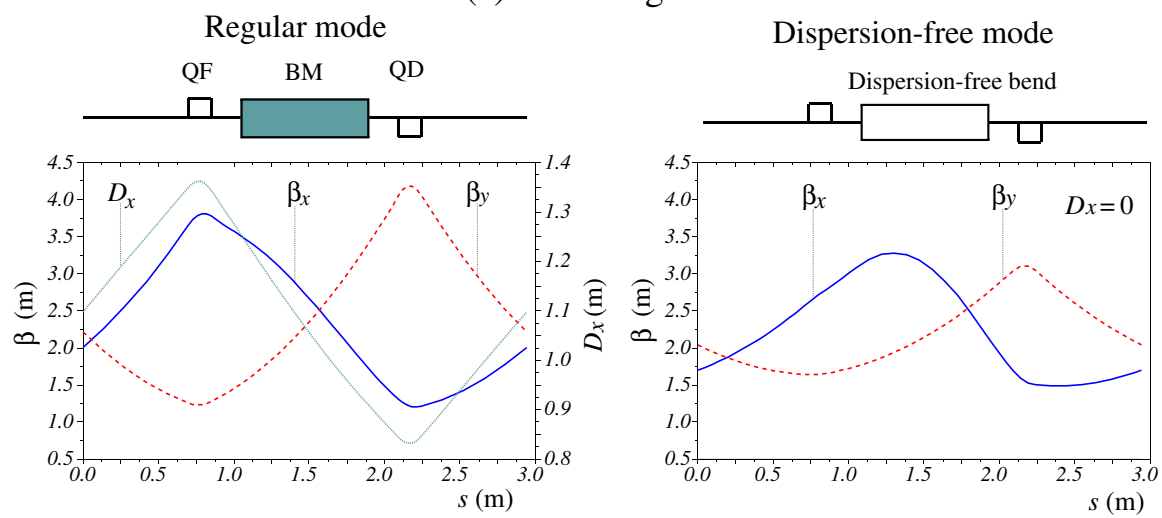

(b) Test Ring II
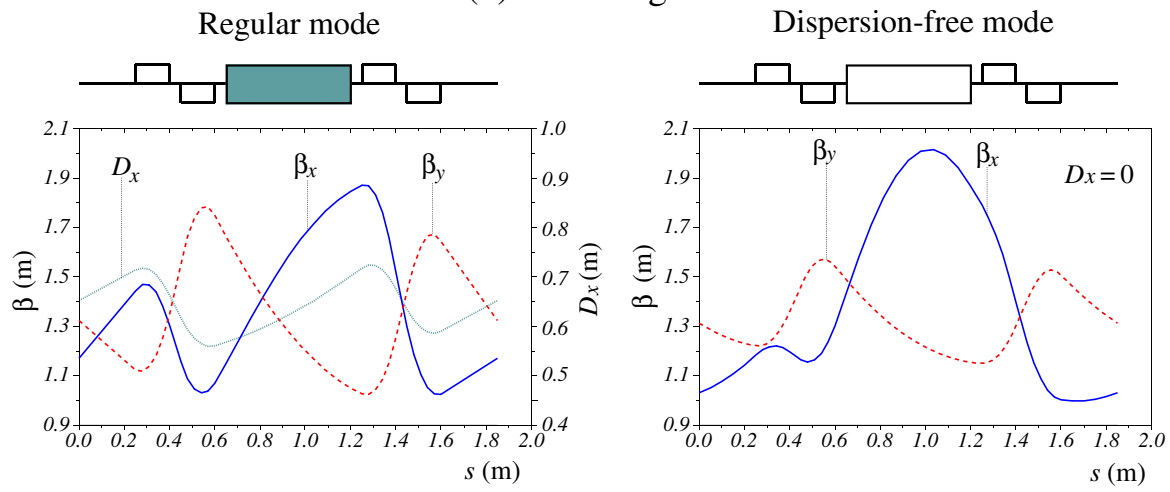

(c) S-LSR

Regular mode
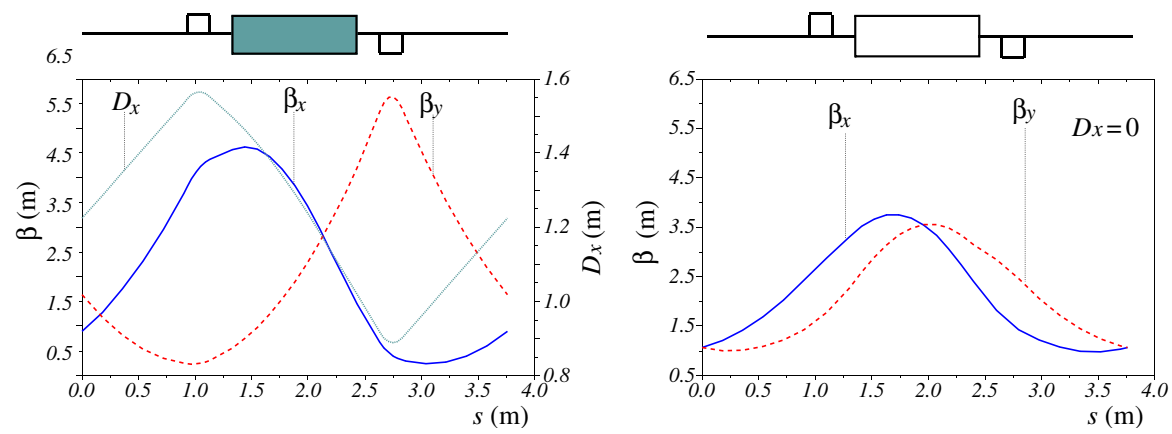

FIG. 1. Lattice functions of three storage rings in the regular operating mode and in the dispersion-free mode. The betatron functions $\beta_{x(y)}$ and the dispersion function $D_{x}$ across a unit cell have been displayed. In the dispersion-free mode, electrostatic deflectors are turned on in all bending regions. The bare betatron tunes have been adjusted at the same numbers in both modes: $\left(\nu_{x}, \nu_{y}\right)=$ $(2.23,2.23)$ for Test Ring I and Test Ring II, and $\left(\nu_{x}, \nu_{y}\right)=(2.07,2.07)$ for S-LSR. Other parameters are summarized in Table I.

TABLE I. Main lattice parameters considered in simulations.

\begin{tabular}{lccc}
\hline \hline \multicolumn{1}{c}{ Lattice } & Test Ring I & Test Ring II & S-LSR \\
\hline Superperiodicity & 10 & 10 & 6 \\
Circumference [m] & 29.4 & 18.5 & 22.557 \\
Typical betatron tunes $\left(\nu_{x}, \nu_{y}\right)$ & $(2.23,2.23)$ & $(2.23,2.23)$ & $(2.07,2.07)$ \\
Transition gamma $\gamma_{t}$ (regular mode) & 2.09 & 2.17 & 1.67 \\
$\quad$ (dispersion-free) & $\infty$ & $\infty$ & $\infty$ \\
Field index of bending magnetic fields & 0 & 0 & 0 \\
Field index of bending electric fields $n$ & 1 & 1 & 0 \\
\hline \hline
\end{tabular}




\section{BEAM CRYSTALLIZATION IN THE DISPERSION-FREE MODE}

\section{A. Formation of multidimensional crystalline beams}

We have performed a number of systematic MD simulations, assuming the lattice structures in Fig. 1. Since no essential difference has been identified so far in the three cases as long as the line density of the beam is relatively low [27], we only show the results on Test Ring I in this subsection. The strength of the untapered friction is set at $f_{x}=f_{y}=0.05$ and $f_{z}=0.10$ that suffice for overcoming the heating from intrabeam scattering. Some examples of final ion distributions after cooling are shown in Fig. 2. The left pictures correspond to the regular operation while the right pictures to the dispersion-free operation. We find that $1 \mathrm{D}$ or $2 \mathrm{D}$ crystals can be attained even in the regular operating mode. At higher line density, it is no longer possible to form an ordered configuration. As expected, the simple untapered force works as a heating source at ultralow temperature and destroys $3 \mathrm{D}$ crystals in the dis- persive environment $[9,16]$. In contrast, any kinds of crystalline states can be reached in the dispersion-free mode without tapered cooling. The situation is unchanged in Fig. 3 where radio-frequency (rf) cavities have been turned on to bunch the beam. We again observe the formation of stable 1D, 2D, and 3D bunched crystals in the dispersionfree mode, but fail to establish 3D crystalline states in the regular operation.

Needless to say, the tapered cooling enables us to generate multidimensional crystalline beams even in an ordinary dispersive ring as confirmed in Fig. 4. It is interesting that the horizontal extent of the coasting crystalline beam in Fig. 4 is greater than that in the case of Fig. 2(b) while the two simulations are based on the same fundamental parameters. This can be understood by recalling the role of the centrifugal force in either operating mode. Once a crystalline state is reached, the centrifugal force defocuses the beam horizontally because all particles must have an identical angular velocity. In the dispersion-free mode, the electrostatic potential of the deflector counteracts the (a) Regular mode
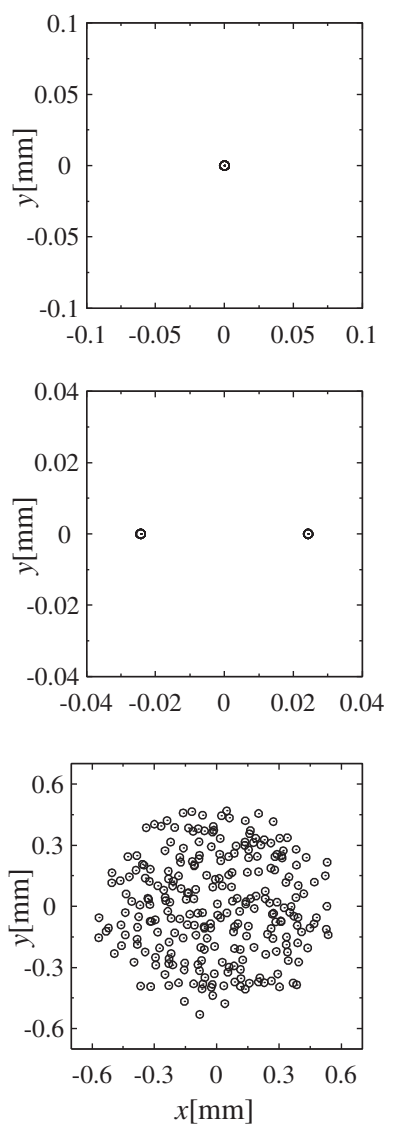
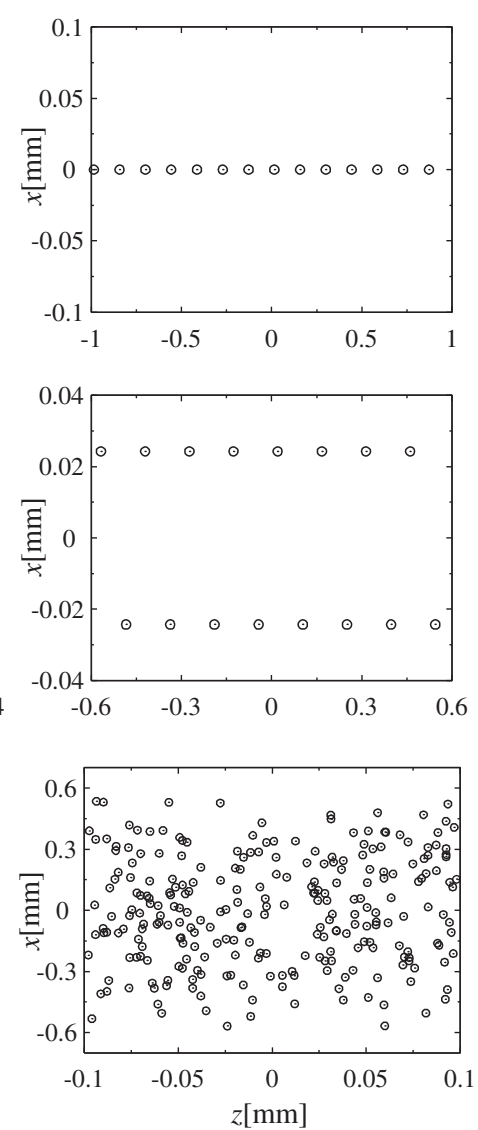

(b) Dispersion-free mode
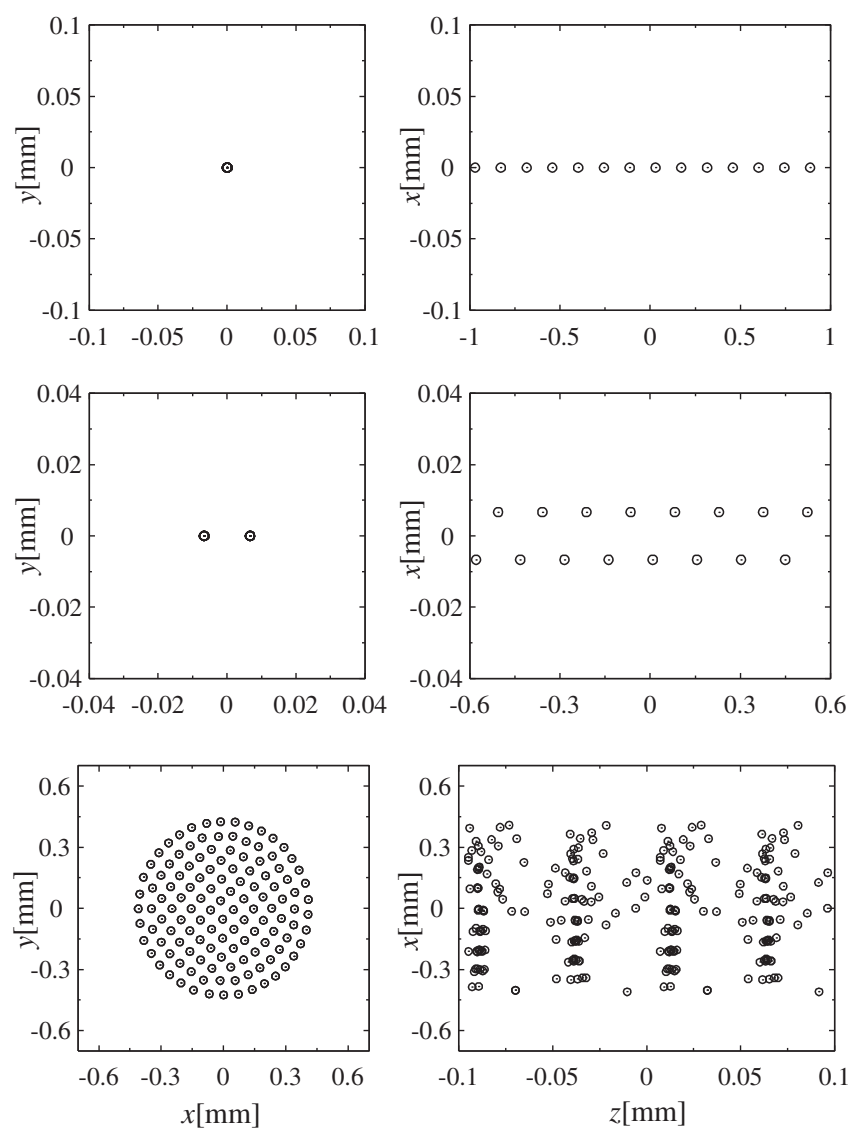

FIG. 2. MD simulation results on Test Ring I with $\left(\nu_{x}, \nu_{y}\right)=(2.23,2.23)$. The spatial configurations of coasting crystalline beams at three different line densities have been depicted. The line density assumed in the top panels is $7.0 \times 10^{3} \mathrm{~m}^{-1}, 1.3 \times 10^{4} \mathrm{~m}^{-1}$ in the middle panels, and $1.3 \times 10^{6} \mathrm{~m}^{-1}$ in the bottom panels. The linear untapered cooling forces with the friction coefficients $f_{x}=f_{y}=$ 0.05 and $f_{z}=0.10$ have been applied to the beams in every lattice period. 
(a) Regular mode
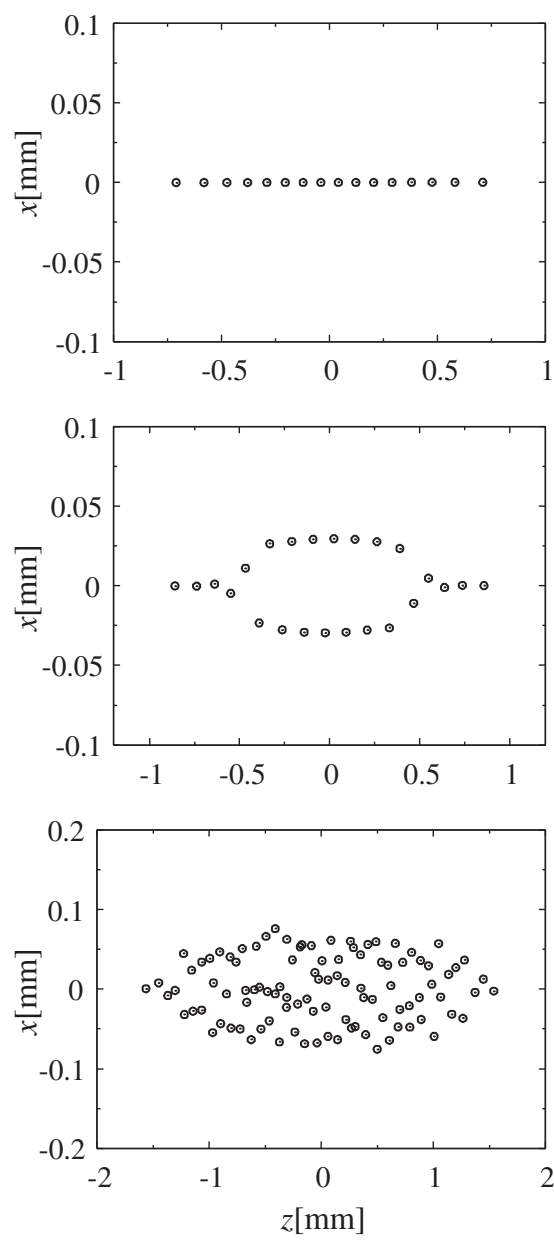

(b) Dispersion-free mode
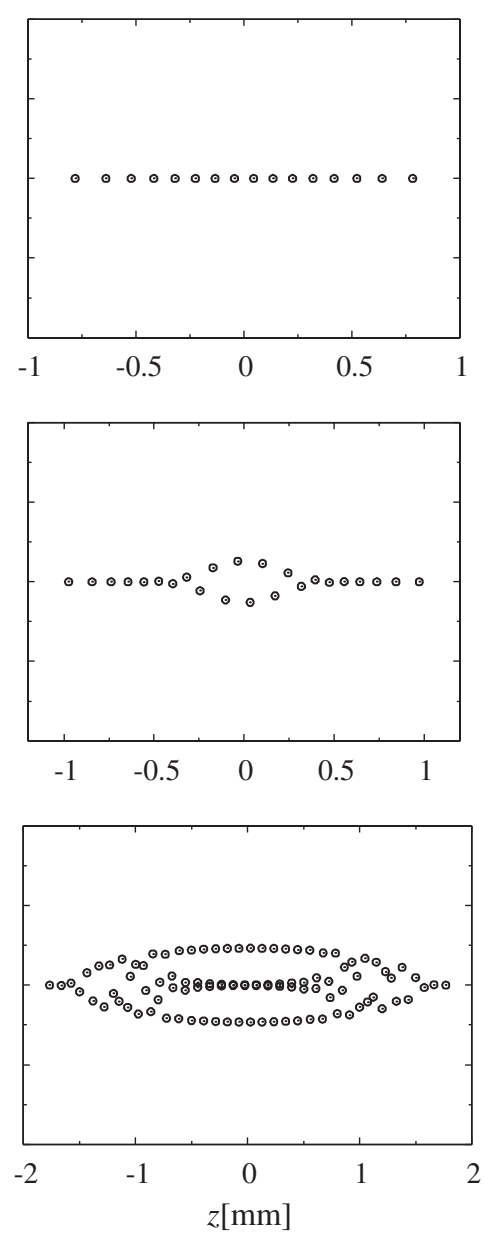

FIG. 3. MD simulation results on Test Ring I. The spatial configurations of bunched crystalline beams have been depicted. The number of ${ }^{24} \mathrm{Mg}^{+}$ions in a bunch is 16 in the top panels, 24 in the middle panels, and 100 in the bottom panels. Ten rf cavities are introduced in the ring to strictly maintain the high lattice symmetry. The rf amplitude and harmonic number of each cavity have been set at $23.23 \mathrm{~V}$ and 100, achieving the synchrotron tune of 0.23 . In the dispersive case, $\mathrm{rf}$ amplitude has been set at $29.45 \mathrm{~V}$ to achieve the same synchrotron tune. Other parameters are identical to those assumed in Fig. 2.

Cross section of coasting beam

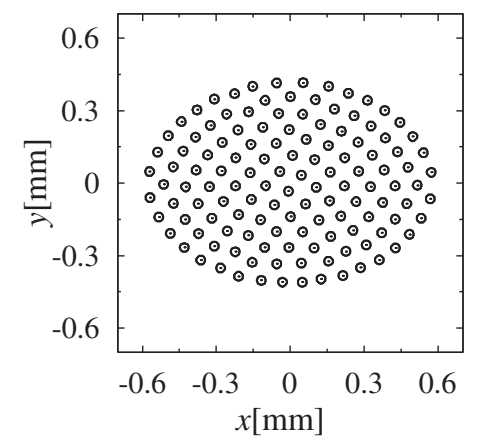

Top view of bunched beam

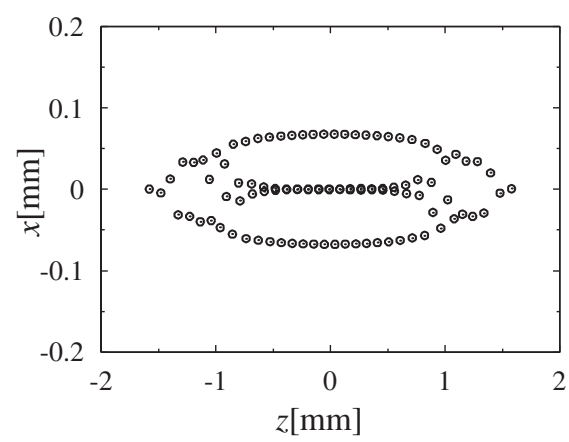

FIG. 4. Examples of multidimensional crystalline beams generated in the regular operating mode of Test Ring I by means of tapered cooling. The friction coefficients assumed here are $f_{x}=f_{y}=0.05$ and $f_{z}=0.10$. The tapering factor has been adjusted to $C_{x s}=$ 0.315 that is evaluated from the theory in Ref. [15]. The line density of the coasting beam is $1.3 \times 10^{6} \mathrm{~m}^{-1}$. The number of ions in a bunch is 100 in the right panel. The synchrotron tune has been set at 0.23 . 


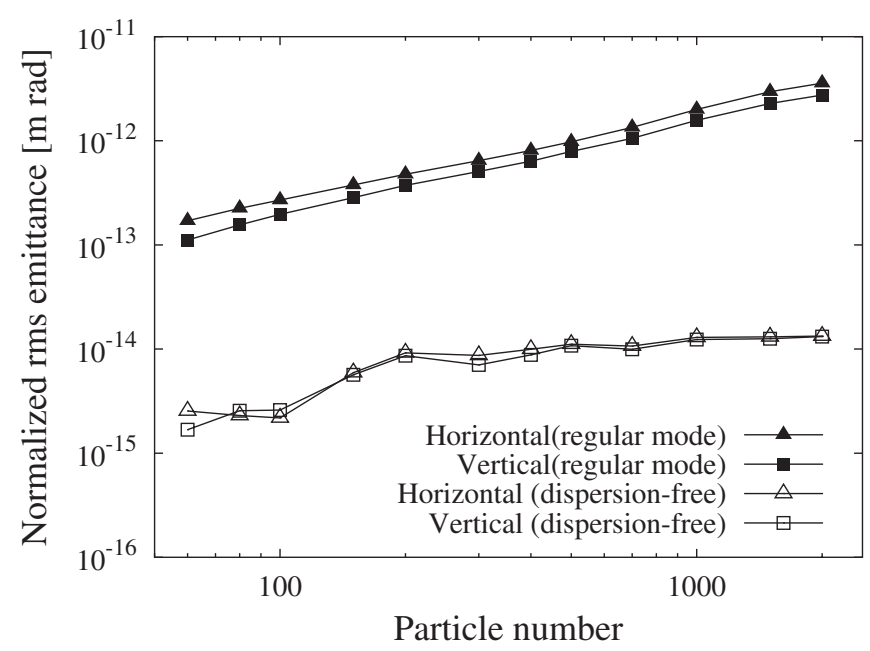

FIG. 5. Final equilibrium transverse emittances accomplished with the untapered linear friction. The abscissa indicates the number of ions in a bunch. The other parameters are identical to those in previous figures. The open marks correspond to dispersion-free cases where achievable emittances are not only much smaller than those in the regular operation cases but also almost independent of the particle number.

defocusing effect as is clear from Eq. (8). The crystal is, therefore, more compressed in the horizontal direction.

The normalized root-mean-squared (rms) emittances of bunched beams cooled with the untapered linear friction have been plotted in Fig. 5. The fundamental lattice parameters are identical to those employed in Fig. 3. We observe a significant difference between the achievable emittances in the two operating modes. In the regular operating mode, the equilibrium emittances linearly increase as the number of ions in a bunch becomes larger, which is consistent to the results of realistic laser cooling simulations in Ref. [16]. The magnitudes of the final emittances are determined by the balance between the external cooling force and internal heating attributed to the shear. If the cooling force is switched off, the beam immediately blows, settling into a much hotter state. On the other hand, no remarkable emittance growth takes place in the dispersion-free mode even after the friction is removed. Unlike the regular operation case, the equilibrium emittances are insensitive to the line density.

\section{B. On the stability of bunched crystalline beams}

As pointed out in previous papers [16,21,28], the dynamic behavior of a bunched Coulomb crystal is much more complicated than that in a Paul ion trap. Since individual particles either gain or lose energy at rf cavities, the transverse motion is seriously affected by the energy modulation through the dispersive coupling, in other words, the cross term $x p_{z}$ in the Hamiltonian. Consequently, even a 1D string crystal oscillates periodically on the horizontal plane. 3D shell crystals also execute horizontal head-tail oscillations that have the same periodic pattern as in the case of 1D strings $[21,28]$. This strongly suggests that rf cavities should be placed symmetrically in every lattice period to stabilize a large bunched crystalline beam. Suppose, for instance, that we have only one cavity sitting in a ring. Then, the horizontal dispersiondriven oscillation has the period equal to the ring circumference, which implies that the lattice periodicity is actually unity, i.e. $N_{\mathrm{sp}}=1$. This makes it impossible to meet the requirement in Eq. (10). In fact, it has been confirmed that we cannot reach a multidimensional crystalline state even with the ideal tapered force unless the lattice sym-

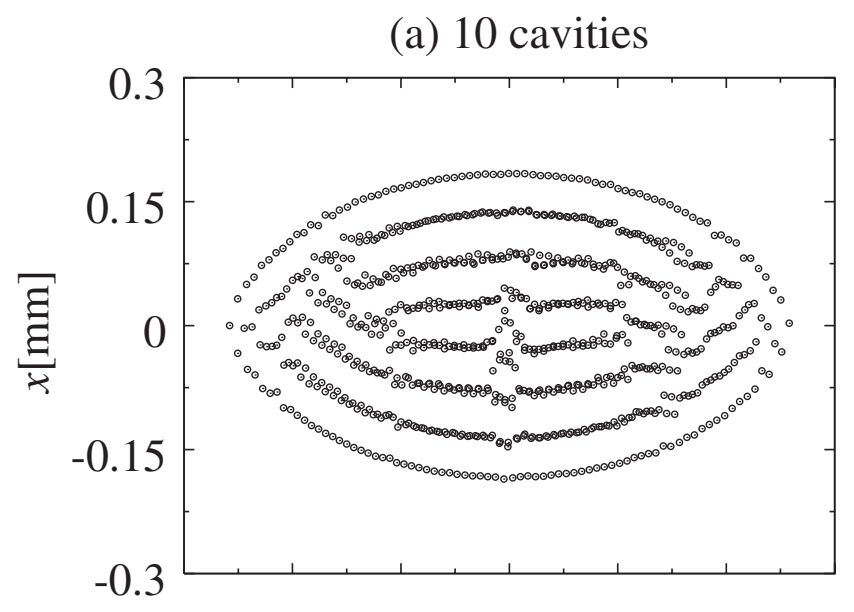

(b) Single cavity

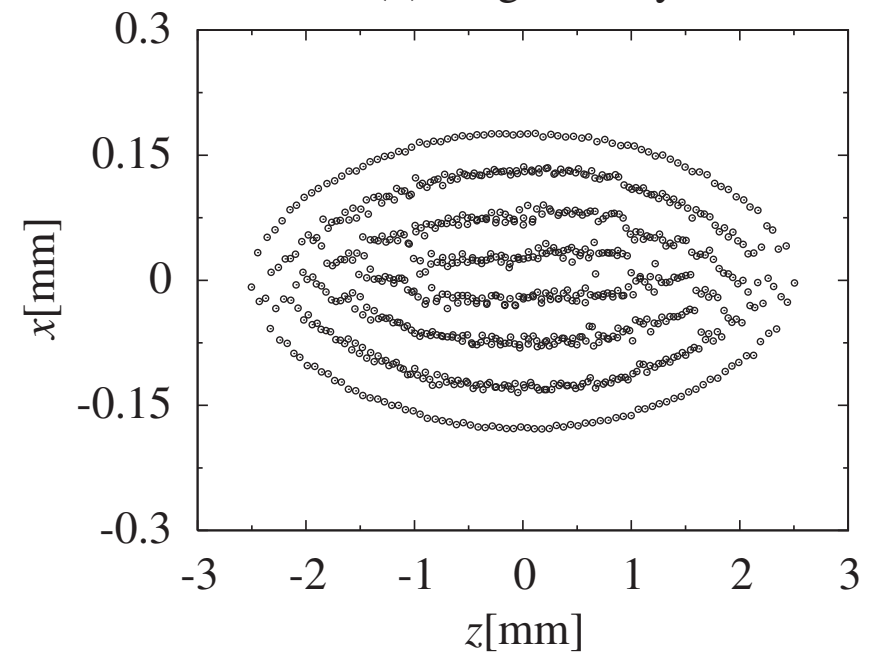

FIG. 6. Bunched 3D crystalline beams produced in Test Ring II at the operating point $\left(\nu_{x}, \nu_{y}, \nu_{z}\right)=(1.46,2.46,0.23)$. The momentum dispersion has been completely suppressed in both cases. We have applied the untapered friction to 800 particles traveling at the kinetic energy of $35 \mathrm{keV}$. In the upper picture, ten rf cavities have been simultaneously excited in order to keep the 10 -fold lattice symmetry, while the ring contains only a single cavity in the lower picture. Although both results look similar, the crystal of the latter case is unstable; namely, it melts in a few hundred turns after the cooling force is removed. 
metry is strictly maintained including the arrangement of rf cavities [16].

An interesting question arises now: what happens in the dispersion-free situation? Is it possible to generate 3D bunched crystals with a single rf cavity excited in the ring? In order to find the answer, we carried out MD simulations assuming the two test storage rings. Figure 6 shows a typical result in which the lattice of Test Ring II operated at $\left(\nu_{x}, \nu_{y}, \nu_{z}\right)=(1.46,2.46,0.23)$ has been taken. The number of ions in a bunch is 800 . As already verified in the last subsection, it is possible to make a stable multishell crystal without tapered cooling when 10 cavities are excited simultaneously [Fig. 6(a)]. In the lower picture, we have shut down 9 of the 10 cavities while keeping the synchrotron tune by increasing the rf voltage. An ordered structure very similar to the upper case has been formed without tapered cooling, which is never anticipated in regular dispersive situations [16]. In both cases, transverse normalized rms emittances less than the order of $10^{-13} \mathrm{~m} \mathrm{rad}$ has been achieved. This is another great advantage in a dispersion-free storage ring, considering the practical difficulty in putting an rf cavity in each lattice period. Similar MD results have been obtained at different operating points and/or with the lattice of Test Ring I. It has, however, turned out that 3D crystalline beams bunched by a single cavity are not completely stable; they melt in a few hundred turns, unlike crystalline bunches in a ring holding perfect high symmetry. Although the Hamiltonian (8) indicates that the longitudinal motion in the dispersion-free mode is decoupled from the transverse motion in a single-particle point of view, we do have the strong Coulomb potential that correlates the 3 degrees of freedom. External forces in the longitudinal direction can, therefore, affect the transverse dynamics of the beam through the Coulomb coupling. The influence is not so strong as in the full dispersive operation, but definitely finite. When the ring includes only one cavity, the period of the longitudinal breathing oscillation is equal to the ring circumference and, accordingly, the beam can recognize that the strict superperiodicity of the ring is unity, i.e. $N_{\mathrm{sp}}=1$. This results in the weak breakdown of the maintenance condition in Eq. (10). Even then, we can prevent the serious emittance growth of a bunched crystalline beam in practice simply by keeping the cooling force on.

\section{STRUCTURAL TRANSITION OF CRYSTALLINE} BEAMS

\section{A. Coasting crystalline beams}

The structure of infinitely long Coulomb crystals in a harmonic potential was studied extensively by Hasse and Schiffer as a model of coasting crystalline beams [4]. It was demonstrated that, as the linear density of particles increases, the crystalline structure changes from 1D chain to $2 \mathrm{D}$ zigzag and finally to $3 \mathrm{D}$ shell structures. The linear densities of various structural transitions are uniquely determined by the normalized density,

$$
\lambda=\zeta a_{\mathrm{wS}},
$$

where $\zeta$ is the linear density of the particles in the rest frame, and $a_{\mathrm{WS}}$ is the Wigner-Seitz radius that corresponds to the radius of the average spherical volume occupied by a particle in a crystalline state. When the beam focusing strengths are equal in both transverse directions, the smooth approximation gives

$$
a_{\mathrm{WS}}=\left(\frac{3 q^{2} R^{2}}{8 \pi \varepsilon_{0} m_{0} \beta^{2} \gamma^{2} c^{2} \nu_{\perp}^{2}}\right)^{1 / 3},
$$

where $R$ is the average radius of the storage ring, $\varepsilon_{0}$ is the dielectric constant in vacuum, and $\nu_{\perp}$ represents the effective transverse tune in the beam rest frame. In a regular dispersive ring, the net radial focusing force received by a crystalline beam is proportional to $\nu_{x}^{2}-\gamma^{2}$ instead of $\nu_{x}^{2}$ [7]. This basically comes from the fact that the centrifugal force in the bending regions works as defocusing in a crystalline state because all particles must have the same angular velocity. Therefore, in order to apply the definition in Eq. (13), the transverse bare tunes should not be equal in both directions but rather chosen such that $\nu_{x}^{2}-\gamma^{2}=$ $\nu_{y}^{2}\left(=\nu_{\perp}^{2}\right)$. Table II summarizes the transition densities identified by MD simulations in which the lattices of Test Ring I and Test Ring II have been assumed. The operating betatron tunes are fixed at $\left(\nu_{x}, \nu_{y}\right)=(2.44,2.23)$ for the regular operating mode and $\left(\nu_{x}, \nu_{y}\right)=(2.23,2.23)$ for the dispersion-free mode (because the net radial focusing is no longer weakened by the centrifugal force after the dispersion is eliminated). As expected, a transition from one structure to another takes place in a dispersion-free ring at the line density close to the theoretical prediction with

TABLE II. Normalized linear densities of structural transitions.

\begin{tabular}{lccccc}
\hline \hline & \multicolumn{3}{c}{ Normalized linear density } & & \\
Crystal structure & Hasse-Schiffer [4] & $\begin{array}{c}\text { Test Ring I } \\
\text { (dispersion-free mode) }\end{array}$ & $\begin{array}{c}\text { Test Ring II } \\
\text { (dispersion-free mode) }\end{array}$ & $\begin{array}{c}\text { Test Ring I } \\
\text { (regular mode) }\end{array}$ & $\begin{array}{c}\text { Test Ring II } \\
\text { (regular mode) }\end{array}$ \\
\hline String (1D) & $0<\lambda<0.709$ & $0<\lambda<0.70$ & $0<\lambda<0.69$ & $0<\lambda<0.65$ & $0<\lambda<0.69$ \\
Zigzag (2D) & $0.709<\lambda<0.964$ & $0.70<\lambda<0.94$ & $0.69<\lambda<0.95$ & $0.65<\lambda<1.1$ & $0.69<\lambda<1.1$ \\
Single shell (3D) & $0.964<\lambda<3.10$ & $0.94<\lambda<3.2$ & $0.95<\lambda<3.2$ & $1.1<\lambda<3.0$ & $1.1<\lambda<3.3$ \\
\hline \hline
\end{tabular}


the harmonic potential model. We also observe reasonable agreement between the theory and simulations even when the dispersion is finite. As far as the present MD results are concerned, it appears that the ring dispersion tends to push down the 1D-to-2D transition threshold while pushing up the 2D-to-3D transition threshold. The amount of the shifts weakly depends on the lattice designs.

\section{B. Bunched crystalline beams}

Coulomb crystals confined in an ion trap are similar to bunched crystalline beams (rather than coasting crystalline beams). Both crystals are compressed by external forces in all three directions and thus spatially localized. The nature of such three-dimensionally bounded Coulomb crystals was theoretically studied by Schiffer [29] and Dubin [30], who considered the simple time-independent uniform confinement of charges. According to their analysis, the structural transitions can be well described by the anisotropy parameter $\alpha \equiv \omega_{z}^{2} / \omega_{r}{ }^{2}$ where $\omega_{z}$ and $\omega_{r}$ denote the strengths of the longitudinal and transverse particle confinement fields. A crystal is more compressed in the longitudinal direction as $\alpha$ increases; we can then convert a $1 \mathrm{D}$ string into a $2 \mathrm{D}$ zigzag crystal without increasing the number of particles in a bunch. This happens also in discrete alternating-gradient (AG) focusing systems [21]. By further increasing $\alpha$, a zigzag can be transformed to a twisted helical structure [29]. These specific transitions are expected to take place at [30]

$$
\alpha_{i}(N)=\left(\frac{8}{3 x_{i} N}\right)^{2}\left[\ln \left(3 x_{i} N / 2^{3 / 2}\right)-1\right], \quad i=1 \text { or } 2,
$$

where $x_{1}=2.05, x_{2}=1.29$, and $N$ is the number of particles in the trap. $\alpha_{1}$ and $\alpha_{2}$ are the threshold of 1D to 2D and that of 2D to 3D transitions, respectively. For example, when $N=100$, Eq. (14) predicts that the transition from string to zigzag occurs at $\alpha_{1}=7.41 \times 10^{-4}$ and the transition from zigzag to helix at $\alpha_{2}=1.67 \times 10^{-3}$.

An analogous argument should apply to bunched beams propagating in storage rings. In that case, the focusing parameters $\omega_{z}$ and $\omega_{r}$ are replaced, respectively, by the synchrotron tune and betatron tunes; namely, we redefine an anisotropy parameter as $\tilde{\alpha} \equiv \nu_{z}^{2} / \nu_{\perp}^{2}$ that is generally much less than unity. Figure 7 shows MD results where the lattice of Test Ring I has been adopted. Owing to the same reason as discussed in the last subsection, the betatron tunes for the regular mode have been fixed at 2.44 in the horizontal direction and 2.23 in the vertical direction (thus, $\nu_{\perp}=2.23$ ). The synchrotron tune $\nu_{z}$ is taken as a variable to change $\tilde{\alpha}$. Ten cavities placed around the ring at equal intervals have the same rf amplitude to ensure the strict lattice symmetry. Tapered cooling has been applied in the regular operating mode. We see that Eq. (14) well explains the structural transitions of $2 \mathrm{D}$ and $3 \mathrm{D}$ crystalline beams in the dispersion-free ring when $N$ is small. In particular,

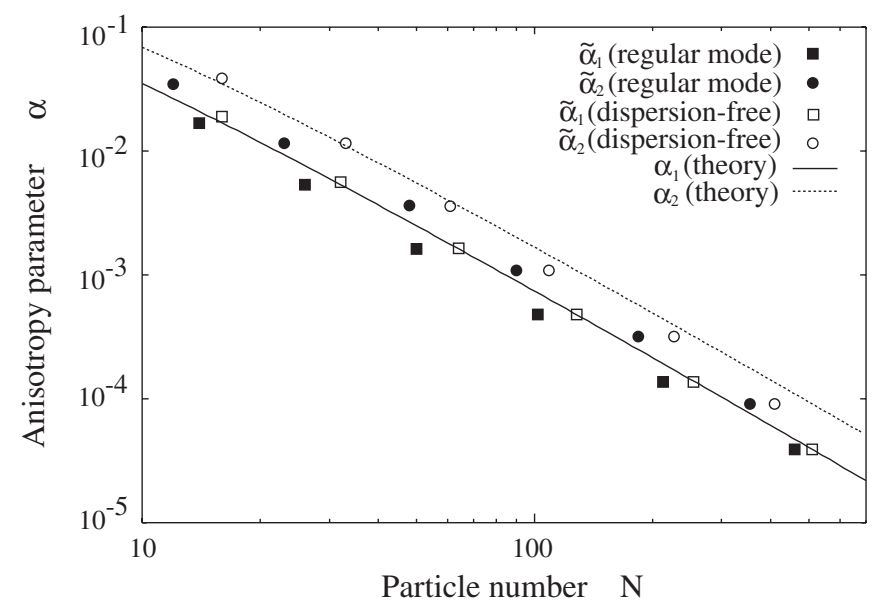

FIG. 7. Anisotropy parameter $\tilde{\alpha}$ of bunched crystalline beams. The two lines represent the boundaries of the 1D-to-2D transition and of the 2D-to-3D transition theoretically predicted by Dubin [Eq. (14)]. The theory says that, in the region below the solid line, the crystalline configuration is the 1D string while it becomes a 3D shell above the dotted line. MD results are indicated with open and filled marks corresponding, respectively, to the dispersion-free and regular operations of Test Ring I.

excellent agreement between the theory and MD simulations has been obtained for $\alpha_{1}$ (1D to 2D transition). With regard to the regular dispersive operation, both types of transitions occur at $\tilde{\alpha}$ smaller than the theoretical prediction in Eq. (14). It is also found that $\tilde{\alpha}_{2}$ tends to be more deviated from the theoretical line as $N$ increases, no matter whether the ring has dispersion or not.

\section{APPROACH TO BEAM ORDERING AT S-LSR}

A possible combination of low transverse tunes at SLSR operated in the regular mode is $\left(\nu_{x}, \nu_{y}\right)=(1.44,1.44)$ that satisfies the condition in Eq. (11). Since the dispersion suppressor enhances the transverse focusing force, the tune exceeds $1.5\left(=N_{\mathrm{sp}} / 4\right)$ in the dispersion-free operation. We then expect serious disturbance of cooling due to linear resonance crossing especially when the line density of the beam is high. It is, however, possible for the ring to satisfy the maintenance condition in the ultralow temperature regime, i.e., Eq. (10), which means that an ordered state could be stable if it is reached somehow. At low line density, it is probably feasible to form a crystal because the growth rates of linear coherent resonances that must be crossed during a cooling process depend on the line density of the beam; the instability is actually weaker at lower density. In order to figure out how much can be done in SLSR, we here simulate cooling at the operating point $\left(\nu_{x}, \nu_{y}\right)=(2.07,2.07)$ that is possible in both regular and dispersion-free modes.

We start from an ideal case where strong 3D cooling forces are available. The friction coefficients are set at $f_{x}=f_{y}=0.05$ and $f_{z}=0.10$. Figure 8 shows the nor- 


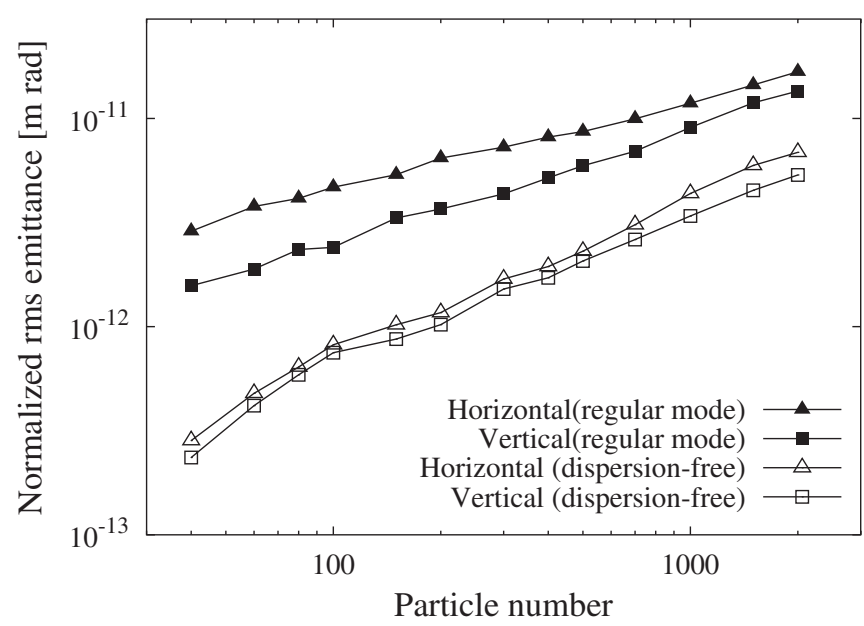

FIG. 8. Normalized rms emittances in the final equilibrium states reached at S-LSR after 3D untapered cooling. The three tunes have been adjusted at $\left(\nu_{x}, \nu_{y}, \nu_{z}\right)=(2.07,2.07,0.22)$. The anisotropy parameter $\tilde{\alpha}$ is the same as in the cases of Figs. 3 and 5. The lattice functions of each operation mode have been plotted in Fig. 1. Other simulation parameters are summarized in Table I.

malized rms emittances achieved in the final equilibrium states. Six rf cavities have been switched on to bunch a $35 \mathrm{keV}{ }^{24} \mathrm{Mg}^{+}$beam. In contrast to the previous MD results in Fig. 5, the equilibrium emittances monotonically increase at higher beam intensity even when the ring dispersion is completely suppressed. The emittances in the dispersion-free mode are more than 2 orders of magnitudes higher than those in Test Ring I. No stable crystalline states have been established even in the dispersion-free operation when the particle number in a bunch exceeds about 40. This suggests that the reachable emittance has been limited by the resonance instability rather than the shear effect in the present case.

Finally, we turn off the transverse cooling force, recalling that laser cooling is effective only in the longitudinal direction. The longitudinal dissipation can be easily extended to the other directions by resonantly coupling the degrees of freedom $[11,12]$. In the dispersion-free operation, a special rf cavity excited in a deflective mode is necessary [31] to develop a linear coupling between the transverse and longitudinal directions. We thus place a coupling cavity in one of the straight sections for horizontal cooling. The dissipative effect can be further brought to the vertical direction through a coupling provided by a solenoid magnet (or a skew quadrupole magnet or a vertical coupling cavity). S-LSR is actually equipped with a solenoid for electron cooling. In order to maximize the indirect cooling efficiencies in the transverse directions, the operating point of the ring must be sufficiently close to linear difference resonances [11,12]; namely, the tunes must be chosen such that $\nu_{x}-\nu_{z} \approx$ integer and $\nu_{x}-\nu_{y} \approx$ integer. The fundamental simulation parameters are listed
TABLE III. Parameters for 3D cooling at S-LSR

\begin{tabular}{lc}
\hline \hline Ion species & ${ }^{24} \mathrm{Mg}^{+}$ \\
Kinetic beam energy & $35 \mathrm{keV}$ \\
Betatron tunes & $(2.07,2.07)$ \\
Synchrotron tune & 0.07 \\
Bending radius & $1.05 \mathrm{~m}$ \\
Field strength of the bending magnets & $0.252 \mathrm{~T}$ \\
Field strength of the electrostatic & $66.7 \mathrm{kV} / \mathrm{m}$ \\
$\quad$ & \\
$\quad$ deflector for dispersion-free operation & 6 \\
$N_{\text {sp }}$ (without the cavities) & $22.557 \mathrm{~m}$ \\
Ring circumference & $0.80 \mathrm{~m}$ \\
Axial length of the solenoid & $40 \mathrm{G}$ \\
Strength of the solenoid field & 100 \\
Harmonic number of the rf cavity & $21.0 \mathrm{~V}$ \\
Amplitude of the rf voltage & 100 \\
Harmonic number of the coupling cavity & $200 \mathrm{~V} / \mathrm{m}$ \\
Amplitude of the coupling rf field & \\
\hline \hline
\end{tabular}

in Table III. Figure 9 shows a spatial configuration of a cooled bunch consisting of 76 ions. We observe the formation of an imperfect but clear 3D ordered structure. The normalized $\mathrm{rms}$ emittances of this beam are $4.44 \times$ $10^{-12} \mathrm{~m} \mathrm{rad}$ in the horizontal directions and $4.23 \times$ $10^{-12} \mathrm{~m} \mathrm{rad}$ in the vertical directions, both of which are much lower than ever recorded in real cooling experiments. As pointed out in Ref. [16], we can never reach such a 3D ordered state in a regular dispersive storage ring with

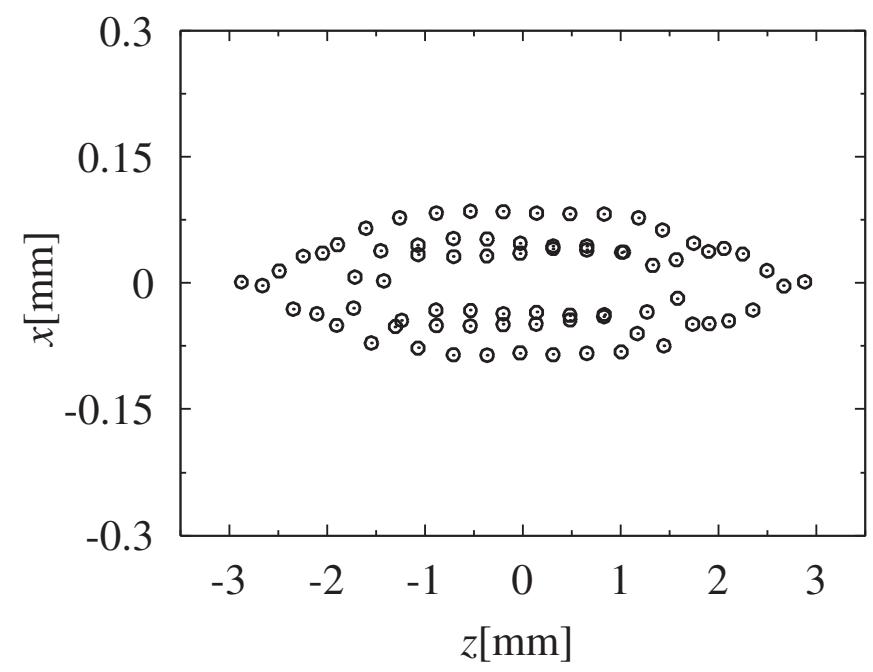

FIG. 9. Spatial configuration (top view) of a bunched beam in S-LSR at the operating point $\left(\nu_{x}, \nu_{y}, \nu_{z}\right)=(2.07,2.07,0.07)$. The momentum dispersion of the ring has been suppressed by the electrostatic deflectors. The simple, untapered linear cooling force with $f_{z}=0.20$ is applied only in the longitudinal direction once in every turn. The transverse cooling has been accomplished by the resonant coupling method based on a special coupling cavity [11] and on a solenoid magnetic field. In order to recover the lattice symmetry, we have switched off the coupling cavity after the cooling is completed. 
realistic cooling. At lower line density, it is certainly possible to generate $1 \mathrm{D}$ string and 2D zigzag crystals.

\section{CONCLUSIONS}

We have demonstrated, through systematic MD simulations, that it is possible to produce multidimensional crystalline beams in a dispersion-free storage ring without relying on the special tapered longitudinal force. Provided that the lattice fulfills the two necessary conditions, i.e., Eqs. (9) and (11), and that the cooling force applied is sufficiently strong, various coasting and bunched crystalline states can be reached with the simplest linear friction. This suggests that the conventional laser cooling technique enables us to generate large 3D crystalline beams, which is impossible in the common dispersive situations. Even when a beam is bunched by a single rf cavity that breaks the strict lattice symmetry, we can form and maintain a multishell ordered state by keeping the cooling force on. MD simulations based on the lattice of S-LSR at Kyoto University have been performed, confirming that $3 \mathrm{D}$ beam ordering can be achieved in the dispersion-free operation without direct transverse cooling (Fig. 9). By exciting not only a regular rf cavity but also a coupling rf cavity, the longitudinal cooling force can be extended to the transverse directions [11].

The structural transitions of crystalline beams in regular and dispersion-free storage rings have also been investigated. It is found that, in the case of coasting beams, the transition line densities in a dispersion-free storage ring agree fairly well with the predictions from the HasseSchiffer theory. On the other hand, we encounter some discrepancy between the theory and MD simulations with regular dispersive lattices. Comparison of transition densities has also been made between bunched crystalline beams and Coulomb crystals in ion traps. The Dubin's formula (14) seems applicable to crystalline beams in a dispersion-free ring. In particular, the threshold of 1D-to2D transition can be predicted precisely by Eq. (14) even when the beam is exposed to spatially inhomogeneous, AG focusing forces. In a regular dispersive ring, a string (zigzag) crystal is transformed to the zigzag (single-shell) configuration at the $\tilde{\alpha}$-value lower than the theoretical expectation.

\section{ACKNOWLEDGMENTS}

The authors are indebted to Dr. J. Wei for useful discussions on the relativistic formalism of the equations of motion in the beam rest frame. One of the authors (M. Ikegami) would like to thank Professor A. Noda for his support in the course of this work.

[1] J. P. Schiffer and P. Kienle, Z. Phys. A 321, 181 (1985).
[2] A. Rahman and J.P. Schiffer, Phys. Rev. Lett. 57, 1133 (1986).

[3] J.P. Schiffer and A. Rahman, Z. Phys. A 331, 71 (1988).

[4] R. W. Hasse and J. P. Schiffer, Ann. Phys. (N.Y.) 203, 419 (1990).

[5] J. Beebe-Wang, N. Elander, and R. Schuch, Nucl. Instrum. Methods Phys. Res., Sect. B 79, 806 (1993).

[6] J. Wei, X.-P. Li, and A. M. Sessler, Phys. Rev. Lett. 73, 3089 (1994).

[7] J. Wei, X.-P. Li, and A. M. Sessler, Brookhaven National Laboratory Report No. BNL-52381, 1993.

[8] J. Wei, A. Draeseke, A.M. Sessler, and X.-P. Li, in Crystalline Beams and Related Issues, edited by D.M. Maletic and A. G. Ruggiero (World Scientific, Singapore, 1996), p. 229.

[9] J. Wei, H. Okamoto, and A. M. Sessler, Phys. Rev. Lett. 80, 2606 (1998).

[10] Recent theoretical studies have revealed that the betatron phase advance per lattice period should be less than 90 degrees in order to avoid strong linear resonance crossing throughout the beam cooling process. See Ref. [25].

[11] H. Okamoto, A. M. Sessler, and D. Möhl, Phys. Rev. Lett. 72, 3977 (1994).

[12] H. Okamoto, Phys. Rev. E 50, 4982 (1994).

[13] H. Okamoto and J. Wei, Phys. Rev. E 58, 3817 (1998).

[14] Note that the tapered force in Eq. (1) cools not only the longitudinal direction but also the horizontal direction. For vertical cooling, we can use the resonant coupling method.

[15] H. Okamoto, Phys. Plasmas 9, 322 (2002).

[16] Y. Yuri and H. Okamoto, Phys. Rev. ST Accel. Beams 8, 114201 (2005).

[17] R. E. Pollock, Z. Phys. A 341, 95 (1991).

[18] W. Henneberg, Ann. Phys. (Berlin) 19, 335 (1934).

[19] W. E. Millett, Phys. Rev. 74, 1058 (1948).

[20] M. Ikegami, A. Noda, M. Tanabe, M. Grieser, and H. Okamoto, Phys. Rev. ST Accel. Beams 7, 120101 (2004).

[21] Y. Yuri and H. Okamoto, Phys. Rev. Lett. 93, 204801 (2004).

[22] C. Møller, The Theory of Relativity (Clarendon Press, Oxford, 1952).

[23] M. Ikegami, in Beam Science and Technology, edited by M. Ikegami (Institute for Chemical Research Kyoto University, Uji, 2006), Vol. 10, p. 26.

[24] X.-P. Li, H. Enokizono, H. Okamoto, Y. Yuri, A. M. Sessler, and J. Wei, Phys. Rev. ST Accel. Beams 9, 034201 (2006).

[25] K. Okabe and H. Okamoto, Jpn. J. Appl. Phys. 42, 4584 (2003).

[26] A. Noda, Nucl. Instrum. Methods Phys. Res., Sect. A 532, 150 (2004).

[27] Owing to the linear resonance instability, S-LSR has trouble producing a multishell crystal when the betatron tunes indicated in Table I are chosen. It is, however, possible to form and maintain crystals at relatively low line densities because the instability is weak.

[28] H. Okamoto, Y. Yuri, and K. Okabe, Phys. Rev. E 67, 046501 (2003).

[29] J.P. Schiffer, Phys. Rev. Lett. 70, 818 (1993).

[30] D. H.E. Dubin, Phys. Rev. Lett. 71, 2753 (1993).

[31] T. Kihara, H. Okamoto, Y. Iwashita, K. Oide, G. Lamanna, and J. Wei, Phys. Rev. E 59, 3594 (1999). 